\title{
Successful management of chemotherapy in a stage IV ovarian cancer patient with chronic renal failure
}

\author{
Shoji Kaku $\cdot$ Rikiya Sano $\cdot$ Yuichiro Nakai $\cdot$ \\ Koichiro Shimoya $\cdot$ Takafumi Nakamura
}

Received: 15 March 2012/ Accepted: 2 July 2012

(C) The Japan Society of Clinical Oncology 2012

\begin{abstract}
Because the number of hemodialysis patients is increasing, chemotherapy will need to be performed on more of these patients in the future. Carboplatin is metabolized by the kidneys and excreted in the urine, so delayed excretion caused by renal dysfunction can lead to severe adverse effects. Here, we report on the successful and safe performance of chemotherapy in a hemodialysis patient with stage IV ovarian cancer, based on alteration of the dialysis time and pharmacokinetic monitoring. A 60-year-old woman on hemodialysis for chronic renal failure was referred to our hospital with a left ovarian tumor and multiple metastases. After surgery, she received adjuvant chemotherapy with paclitaxel and carboplatin on a weekly basis. Initially, hemodialysis was done the next morning ( $16 \mathrm{~h}$ after chemotherapy), and grade 3 neutropenia occurred on day 8 of her first course. Pharmacokinetic analysis revealed the persistence of a high total blood platinum concentration until the start of hemodialysis. Therefore, hemodialysis was performed only $2 \mathrm{~h}$ after the second course of chemotherapy. At that time, the total platinum concentration had decreased to the range found in patients with normal renal function, and adverse effects were mild. For the third to sixth courses, chemotherapy was performed safely on an outpatient basis. Her multiple metastases were eliminated by chemotherapy. At 14 months after surgery, there is no evidence of recurrence.
\end{abstract}

This article won the excellent subject prize at the 49th Annual Meeting of the Japan Society of Clinical Oncology.

S. Kaku $(\bowtie) \cdot$ R. Sano · Y. Nakai · K. Shimoya · T. Nakamura Department of Obstetrics and Gynecology, Kawasaki Medical School, 577 Matsushima, Kurashiki, Okayama 701-0192, Japan e-mail: shoji.kaku@gmail.com
Keywords Carboplatin $\cdot$ Hemodialysis - Ovarian cancer

\section{Introduction}

In recent years, the number of patients on hemodialysis for chronic renal failure has been increasing. Accordingly, it is expected that performing chemotherapy in patients on hemodialysis will become more common in the future. However, the optimum method of chemotherapy for hemodialysis patients has not attracted sufficient attention, so the appropriate drug dosage and the correct interval between the administration of anticancer agents and the initiation of dialysis have not been established.

The combination of paclitaxel and carboplatin (TC therapy) is a standard chemotherapy regimen for gynecological cancer. Paclitaxel is metabolized by the liver, and is largely excreted via the alimentary canal in the bile, with only $5-10 \%$ being excreted via the kidneys. Accordingly, it has been reported that even patients with renal failure can be administered paclitaxel at the usual dosage $[1,2]$. In contrast, platinum compounds like cisplatin and carboplatin are metabolized by the kidneys and excreted in the urine. Therefore, delayed excretion of these drugs in patients with renal dysfunction may cause severe adverse reactions. When patients with chronic renal failure are administered cisplatin, it is recommended that hemodialysis should be initiated at the same time or within $1 \mathrm{~h}$ of the start of infusion [3]. When patients with chronic renal failure are administered carboplatin, its half-life has been reported to be $13-16 \mathrm{~h}$, which is about $10 \mathrm{~h}$ longer than in patients with normal renal function $[4,5]$.

The interval between the completion of carboplatin infusion and the initiation of hemodialysis has been discussed by several authors [1, 6-13], but there are few 
reports on the successful management of advanced gynecological cancer in hemodialysis patients. Here, we report a hemodialysis patient in whom chemotherapy was performed safely through the early initiation of dialysis and pharmacokinetic monitoring. Even though this patient had stage IV ovarian cancer, we were able to successfully complete TC therapy with carboplatin and paclitaxel, achieving a complete response.

\section{Case report}

A 60-year-old Japanese woman with chronic renal failure due to sarcoidosis had been on regular hemodialysis three times a week for two years. She complained of abdominal pain and ultrasonography suggested ovarian cancer, so she was referred to our hospital. Based on the results of fluorine-18 fluorodeoxyglucose positron emission tomography/ computed tomography (FDG-PET/CT) and magnetic resonance imaging (MRI), a malignant left ovarian tumor was diagnosed together with right ovarian metastasis, hepatic metastasis, splenic metastasis, and mediastinal lymph node metastases (Fig. 1a). Laboratory tests at the time of hospitalization revealed a white blood cell (WBC) count of $7,040 / \mathrm{Ul}$, hemoglobin of $11.5 \mathrm{~g} / \mathrm{dl}$, platelet count of 164,000/Ul, blood urea nitrogen (BUN) of $42 \mathrm{mg} / \mathrm{dl}$ before hemodialysis versus $11 \mathrm{mg} / \mathrm{dl}$ after hemodialysis, and creatinine (CRE) of $6.47 \mathrm{mg} / \mathrm{dl}$ before hemodialysis versus $2.23 \mathrm{mg} / \mathrm{dl}$ after hemodialysis. Tumor marker levels were as follows: CA125 was $241 \mathrm{U} / \mathrm{ml}, \mathrm{CA} 19-9$ was $170.5 \mathrm{U} / \mathrm{ml}$, and CEA was $2.6 \mathrm{ng} / \mathrm{ml}$. On examination, the patient's height was $152.0 \mathrm{~cm}$, body weight was $42.0 \mathrm{~kg}$, vital signs were normal, and performance status was 0 .

At operation, the left ovary was enlarged and the tumor had spread to involve the right ovary, but there was no ascites in the abdominal cavity. Hysterectomy, bilateral oophorectomy, and partial omentectomy were performed. The pathological diagnosis was serious adenocarcinoma of the ovary in FIGO stage IV (pT1cNxM1).

Because the patient was on hemodialysis, weekly TC therapy (days 1, 8, and 15) was selected postoperatively. Paclitaxel was infused intravenously at $75 \mathrm{mg} / \mathrm{m}^{2}$ (total dose of $100 \mathrm{mg}$ ) over $2 \mathrm{~h}$, followed by intravenous infusion of $50 \mathrm{mg}$ of carboplatin over $1 \mathrm{~h}$. The carboplatin dose was calculated by Calvert's formula [14] to achieve a target area under the concentration versus time curve (AUC) of $2.0 \mathrm{mg} / \mathrm{ml} \mathrm{min}$, with the glomerular filtration rate being set to zero because the patient had no residual renal function.

At first, the patient refused to receive chemotherapy and undergo hemodialysis on the same day, so we decided to perform dialysis at $16 \mathrm{~h}$ after the completion of chemotherapy (on the next morning), along with pharmacokinetic monitoring. Blood was collected just after the completion of carboplatin infusion ( $1 \mathrm{~h}$ after the completion of paclitaxel infusion), as well as $2 \mathrm{~h}(3 \mathrm{~h})$ after, $6 \mathrm{~h}(7 \mathrm{~h})$ after, $12 \mathrm{~h}(13 \mathrm{~h})$ after, and $24 \mathrm{~h}(25 \mathrm{~h})$ after. Blood samples were centrifuged at $3,000 \mathrm{rpm}$ at $4{ }^{\circ} \mathrm{C}$ for $5 \mathrm{~min}$ to obtain plasma to measure the total platinum and paclitaxel concentrations. Paclitaxel was measured by high-performance liquid chromatography (Shimazu 20A, Tokyo, Japan), and total platinum was measured by atomic absorption spectrometry (Hitachi Z-5700, Tokyo, Japan). Blood tests were
Fig. 1 Preoperative and postoperative FDG-PET/CT findings. a Preoperative FDGPET/CT findings. There is accumulation suggesting metastasis in the bilateral ovaries, liver, spleen, and mediastinal lymph nodes (red arrows). Accumulation in the right arm indicates inflammation of the patient's shunt. b Postoperative FDG$\mathrm{PET} / \mathrm{CT}$ findings. There is no evidence of residual metastasis or recurrence
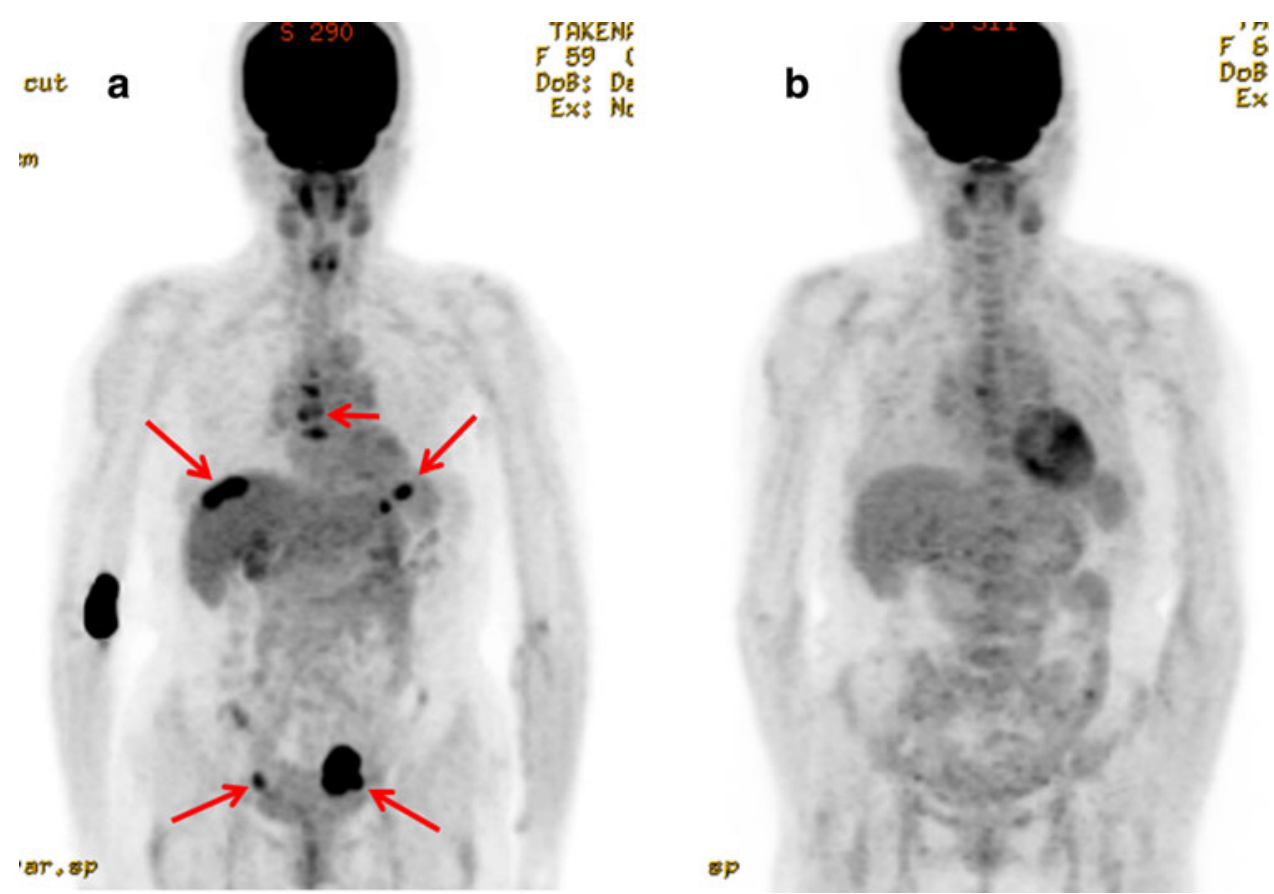
done every 2 days, and the patient was monitored for adverse effects, which were evaluated according to the National Cancer Institute common toxicity criteria (v.4.0).

Grade 3 neutropenia occurred on day 8 of the first course of TC therapy. Pharmacokinetic analysis revealed that a high total platinum concentration had persisted until hemodialysis was performed $16 \mathrm{~h}$ after chemotherapy (Fig. 2a). We thought that the risk of opportunistic infection was higher than usual because our patient had been on steroid therapy for sarcoidosis. After a discussion, the patient agreed to continue the chemotherapy but to undergo hemodialysis $2 \mathrm{~h}$ after the completion of administration. With this new regimen, the blood level of total platinum declined immediately after hemodialysis (Fig. 2a). Because of a concern that the total platinum level had been reduced too rapidly, we obtained approval to measure blood levels in a patient with normal renal function $(\mathrm{GFR}=95 \mathrm{ml} / \mathrm{min}$ ) who had similar physical features (height of $154.0 \mathrm{~cm}$, body weight of $41.0 \mathrm{~kg}$, normal vital signs, and a performance status of 0 ) and who was also receiving weekly TC therapy according to the same schedule: paclitaxel at $75 \mathrm{mg} / \mathrm{m}^{2}$ (total dose of $100 \mathrm{mg}$ ) and carboplatin at an AUC of $2.0 \mathrm{mg} / \mathrm{ml} \mathrm{min} \mathrm{(total} \mathrm{dose} \mathrm{of}$ $240 \mathrm{mg}$ ). We found that the profile of carboplatin in the patient with normal renal function resembled that in our hemodialysis patient (Fig. 2a).

The maximum plasma concentration $\left(C_{\max }\right)$ and AUC of total platinum were, respectively, 2,360 $\mathrm{ng} / \mathrm{ml}$ and $2.39 \mathrm{mg} /$ $\mathrm{ml}$ min during the first course of TC therapy (with hemodialysis at $16 \mathrm{~h}$ after the completion of chemotherapy), while $C_{\max }$ was $2,230 \mathrm{ng} / \mathrm{ml}$ and AUC was $1.75 \mathrm{mg} / \mathrm{ml}$ min during the second course with hemodialysis at $2 \mathrm{~h}$ after chemotherapy. In contrast, paclitaxel showed similar blood concentration profiles during the first and second courses of TC therapy in both our patient on hemodialysis and the patient with normal renal function (Fig. 2b).

With the second course of TC therapy, the adverse effects were grade 2 alopecia, grade 1 nausea, and grade 2 neutropenia, and treatment was completed on an outpatient basis. After six courses of TC therapy, FDG-PET/CT no longer revealed abnormal uptake by the liver, spleen, and mediastinal lymph nodes (Fig. 1b). In addition, tumor marker levels decreased to the normal range. At 14 months after surgery, there is no evidence of recurrence.

\section{Discussion}

Advances in hemodialysis have improved the prognosis of patients with renal failure. As a result, the number of patients on hemodialysis for chronic renal failure has been increasing, especially in Japan, where transplantation remains problematic. Accordingly, more patients are undergoing chemotherapy while on dialysis.
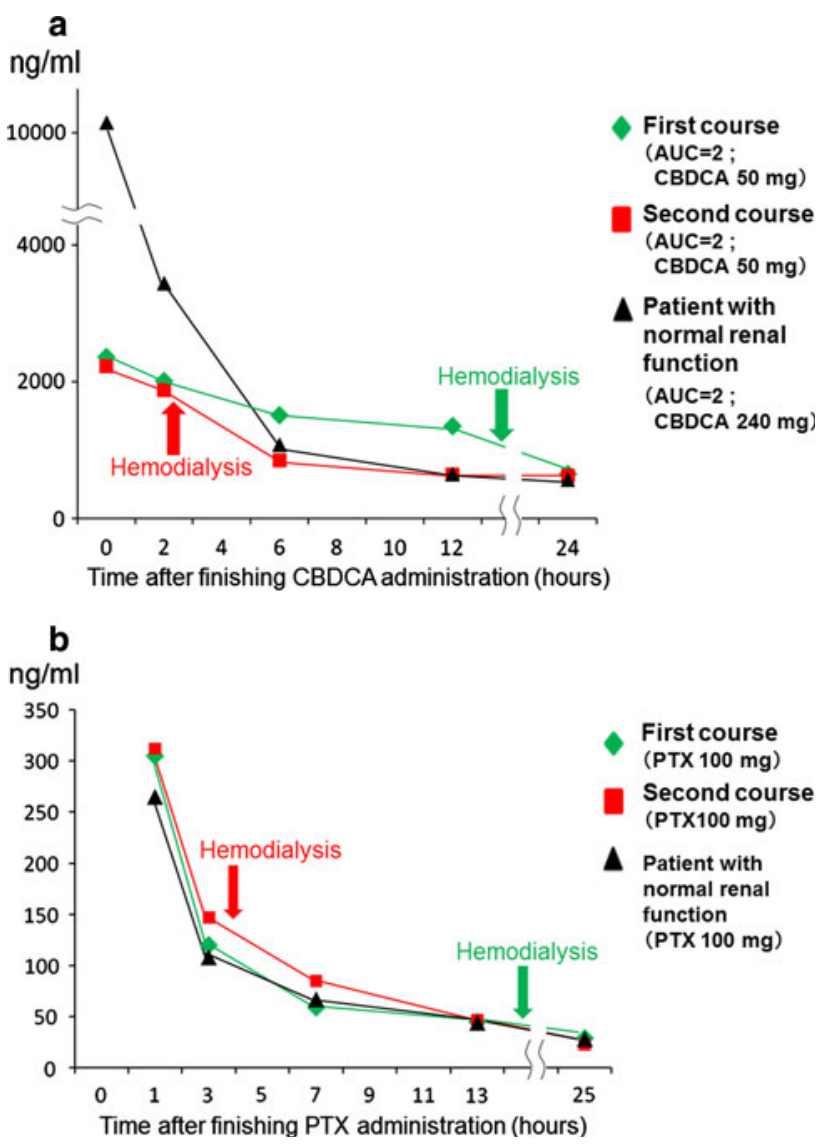

Fig. 2 Profiles of total platinum and paclitaxel (PTX) during the first course of TC therapy for our patient, the second course of TC therapy for our patient, and in a patient with normal renal function. Green arrow: initiation of hemodialysis after the first course of TC therapy; red arrow: initiation of hemodialysis after the second course of TC therapy. $C B D C A$ carboplatin. a Profile of total platinum. b Profile of PTX

Generally, patients with renal failure can be administered paclitaxel at the standard dosage because it is metabolized by the liver and largely excreted in the bile, with only $5-10 \%$ excreted renally $[1,2]$. With regard to carboplatin, the standard dosage has been administered to some patients receiving hemodialysis without severe side effects [15]. On the other hand, the duration of myelosuppression, which is a dose-limiting factor, was prolonged in other patients [10]. Generally, the blood platinum level will remain high until dialysis, and an increased AUC of platinum can lead to various side effects. Table 1 summarizes the reported cases of ovarian cancer with renal failure treated by TC or weekly TC therapy. In previous reports, the appropriate interval between the completion of carboplatin administration and the initiation of hemodialysis has been suggested to range from 0.5 to $24 \mathrm{~h}[1,6-13]$.

In the present case, hemodialysis was initially done at $16 \mathrm{~h}$ after completion of chemotherapy, and a high total platinum concentration persisted until dialysis, resulting in a 


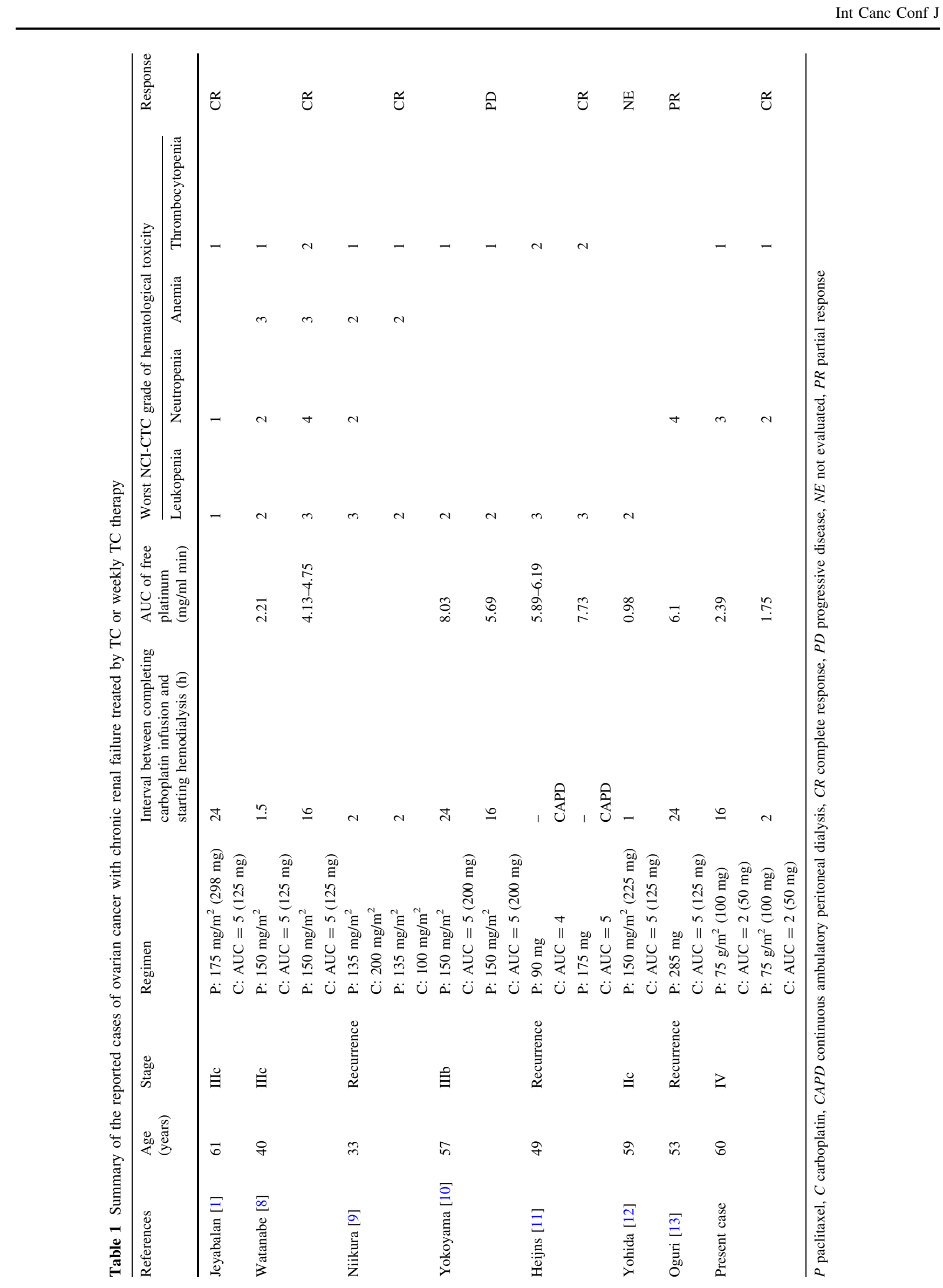

Springer 
large AUC and grade 3 neutropenia on day 8 of the first course. In contrast, when hemodialysis was done $2 \mathrm{~h}$ after the completion of the second chemotherapy course, the total platinum level declined rapidly and the AUC became smaller, with the blood platinum profile resembling that in a patient with normal renal function. As a result, neutropenia was only grade 2 after the second course of TC therapy.

There have been few previous reports on the effect of altering the timing of hemodialysis in the same patient, and few comparisons of the blood levels of chemotherapy agents between patients on dialysis and patients with normal renal function. There have also been few previous assessments of the activity of TC therapy in dialysis patients or the adverse effect profile of this regimen. In the present case, TC therapy was safely completed by altering the timing of dialysis and by monitoring blood platinum levels.

The tumor responded because it was chemosensitive and because we reduced the AUC of platinum and controlled side effects by changing the timing of dialysis so that chemotherapy could be completed as scheduled. Comparison with a patient who had normal renal function showed that early dialysis did not reduce the levels of anticancer drugs excessively, and complete response of the stage IV ovarian cancer was obtained.

In conclusion, performing hemodialysis $2 \mathrm{~h}$ after the completion of TC therapy allowed safe and effective treatment of advanced ovarian cancer. Our experience may be useful to consider when managing other hemodialysis patients who require chemotherapy.

Acknowledgments We thank Yangsil Chang, M.D. and Tsuyoshi Ishida, M.D., who facilitated our work in this case report.

Conflict of interest The authors declare that they have no conflict of interest.

\section{References}

1. Jeyabalan N, Hirte HW, Moens F (2000) Treatment of advanced ovarian carcinoma with carboplatin and paclitaxel in a patient with renal failure. Int J Gynecol Cancer 10:463-468
2. Tomita M, Kurata H, Aoki Y et al (2001) Pharmacokinetics of paclitaxel and cisplatin in a hemodialysis patient with recurrent ovarian cancer. Anticancer Drugs 12:485-487

3. Reidenberg MM (1977) The binding of drugs to plasma proteins and the interpretation of measurements of plasma concentrations of drugs in patients with poor renal function. Am J Med 62: $466-470$

4. van der Vijgh WJ (1991) Clinical pharmacokinetics of carboplatin. Clin Pharmacokinet 21:242-261

5. Chatelut E, Rostaing L, Gualano V et al (1994) Pharmacokinetics of carboplatin in a patient suffering from advanced ovarian carcinoma with hemodialysis-dependent renal insufficiency. Nephron $66: 157-161$

6. Motzer RJ, Niedzwiecki D, Isaacs M et al (1990) Carboplatinbased chemotherapy with pharmacokinetic analysis for patients with hemodialysis-dependent renal insufficiency. Cancer Chemother Pharmacol 27:234-238

7. Inoue A, Saijo Y, Kikuchi T et al (2004) Pharmacokinetic analysis of combination chemotherapy with carboplatin and etoposide in small-cell lung cancer patients undergoing hemodialysis. Ann Oncol 15:51-54

8. Watanabe M, Aoki Y, Tomita M et al (2002) Paclitaxel and carboplatin combination chemotherapy in a hemodialysis patient with advanced ovarian cancer. Gynecol Oncol 84:335-338

9. Niikura H, Koizumi T, Ito K et al (2003) Carboplatin-based chemotherapy in patients with gynecological malignancies on long-term hemodialysis. Anticancer Drugs 14:735-738

10. Yokoyama Y, Futagami M, Higuchi T et al (2006) Pharmacokinetic analysis of paclitaxel and carboplatin in a patient with advanced ovarian cancer during hemodialysis—case report. Eur J Gynaecol Oncol 27:437-439

11. Heijns JB, van der Burg ME, van Gelder T et al (2008) Continuous ambulatory peritoneal dialysis: pharmacokinetics and clinical outcome of paclitaxel and carboplatin treatment. Cancer Chemother Pharmacol 62:841-847

12. Yoshida H, Sumi T, Abe K (2009) Pharmacokinetics of paclitaxel and carboplatin in a hemodialysis patient with advanced ovarian cancer. Eur J Gynaecol Oncol 30:583-585

13. Oguri T, Shimokata T, Inada M (2010) Pharmacokinetic analysis of carboplatin in patients with cancer who are undergoing hemodialysis. Cancer Chemother Pharmacol 66:813-817

14. Calvert AH, Newell DR, Gumbrell LA et al (1989) Carboplatin dosage: prospective evaluation of a simple formula based on renal function. J Clin Oncol 7:1748-1756

15. Yanagawa H, Takishita Y, Bando H et al (1996) Carboplatinbased chemotherapy in patients undergoing hemodialysis. Anticancer Res 16:533-535 